\title{
Análisis comparativo de la implementación de la educación ambiental en programas de licenciatura de tres universidades
}

Comparative Analysis of the Implementation of Environmental Education in Degree Programs of Three Universities

Análise comparativa da implementação da educação ambiental em programas de graduação de três universidades

\author{
Ramón Bedolla Solano \\ Universidad Autónoma de Guerrero, México \\ rabedsol@hotmail.com \\ https://orcid.org/0000-0001-6219-4953 \\ Adriana Miranda Esteban \\ Universidad Autónoma de Guerrero, México \\ mar86_05@hotmail.com \\ https://orcid.org/0000-0003-4313-6146 \\ Oscar Sánchez Adame \\ Universidad Autónoma de Guerrero, México \\ msposcar@hotmail.com \\ https://orcid.org/0000-0001-5202-3835 \\ *Juan José Bedolla Solano \\ jjosebedolla@hotmail.com \\ https://orcid.org/0000-0001-6999-8823
} Instituto Tecnológico de Acapulco, Tecnológico Nacional de México, México

*Autor de correspondencia 


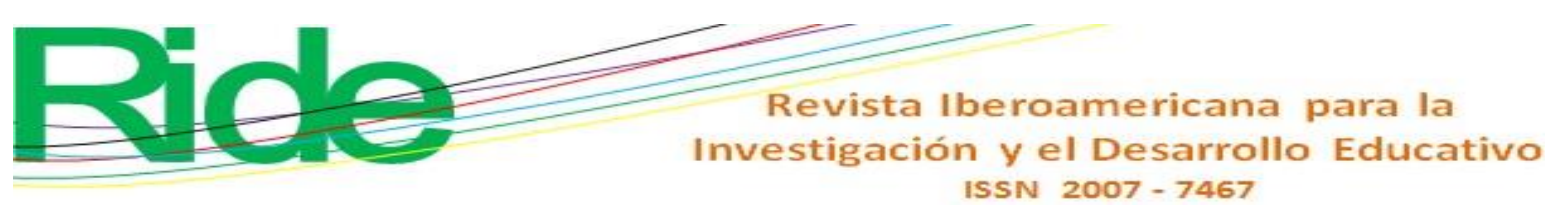

Abstract

The environmental crisis is of great concern throughout the world. In the face of this, environmental education (EE) arises as a process that promotes socio-environmental competences. Educational institutions such as the university represent an adequate space to introduce environmental knowledge into the curriculum, in such a way that the specific competencies and graduation of the educational project are complemented environmentally. The objective of this research was to perform a comparative analysis on the integration of $\mathrm{EE}$ in degree programs of three universities: Autonomous University of Guerrero (Mexico), Regional University of Blumenau (Brazil) and Nelson Mandela Metropolitan University (South Africa).

It is a mixed research with a comparative and descriptive approach. It was carried out in the period 2015-2017. A survey was applied to professors and students in the programs under study, an interview in the programs of Mexico and South Africa and questionnaires of opinion to key informants of the curriculum in the programs of the university of Brazil, and some official documents were reviewed. The dimensions considered in the instruments and techniques were based on the following elements: perception of environmental issues, the relationship between EE and the curriculum, environmental competencies promoted by teachers and students, the revision of regulations that establish the integration of the EE in the study plan.

The result of the survey showed, among other things, that students and professors of educational programs in Mexico have a lower degree of perception of environmental problems compared to the professors of the programs of the other two universities. From the information provided by key informants it was deduced that two programs of the university of Mexico and the two of Brazil contemplate EE in some way. However, in the first, it is in a lesser proportion and in a disciplined way; in the second it is in greater proportion: its integration is transverse and is in process. In the program of South Africa, the EE does not appear. Regarding the official documents, the programs of Mexico and Brazil have a support that supports the integration of the EE; South Africa does not have an official document.

The incorporation of the EE in the educational programs of the universities is glimpsed when the environmental dimension curricularly intervenes in a disciplinary, transversal or other way. Consequently, diverse tasks are developed to preserve the environment and seek sustainability.

Keywords: environmental competences, environmental crisis, curriculum, universities. 


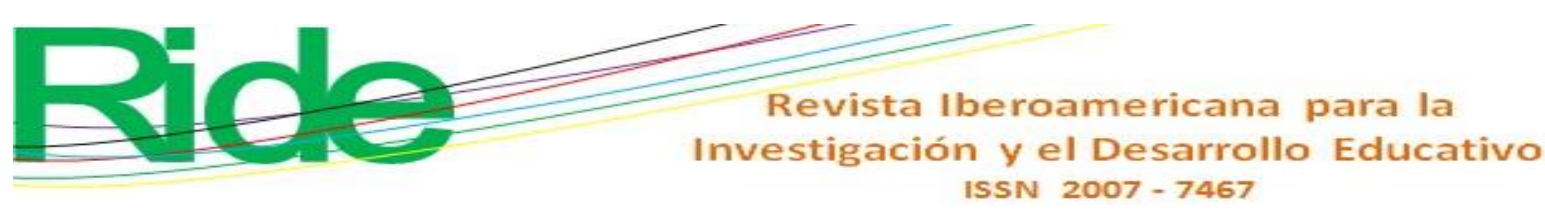

\section{Resumo}

A educação ambiental (EA) surge de grande preocupação em todo o mundo diante da crise ambiental e faz parte de um processo que promove competências socioambientais. A universidade representa um espaço adequado para introduzir conhecimento ambiental no currículo; de tal maneira que as competências específicas e de graduação do projeto educacional sejam complementadas ambientalmente. $\mathrm{O}$ objetivo desta pesquisa foi realizar uma análise comparativa da integração da EA em programas de graduação de três universidades: Universidade Autônoma de Guerrero (México), Universidade Regional de Blumenau (Brasil) e Universidade Metropolitana Nelson Mandela (África do Sul). Trata-se de uma pesquisa mista, com abordagem comparativa e descritiva. Foi realizado no período 2015-2017. Foi aplicada uma pesquisa aos professores e alunos dos programas em estudo, uma entrevista com membros dos programas do México e da África do Sul e questionários de opinião aos principais informantes do currículo dos programas da Universidade do Brasil, e alguns documentos oficiais foram revisados . As dimensões consideradas nos instrumentos e técnicas foram baseadas nos seguintes elementos: percepção do problema ambiental, relação entre a $\mathrm{AE}$ e o currículo, competências ambientais promovidas por professores e alunos e revisão de regulamentos que estabelecem a integração da EA no currículo. O resultado da pesquisa mostrou, entre outras coisas, que estudantes e professores dos programas educacionais do México têm um menor grau de percepção do problema ambiental em comparação com os professores dos programas das outras duas universidades. A partir das informações fornecidas pelos informantes-chave, deduziu-se que dois programas da Universidade do México e os dois do Brasil contemplam, de alguma forma, o EA. No entanto, no primeiro, é em menor proporção e de maneira disciplinar; no segundo está em maior proporção: sua integração é transversal e está em processo. No programa da África do Sul, a EA não figura. Quanto aos documentos oficiais, os programas do México e do Brasil contam com um suporte que apóia a integração da EA; No caso da África do Sul, não há documento oficial. A incorporação da EA nos programas universitários é prevista quando a dimensão ambiental no currículo interfere de maneira disciplinar, transversal ou outra. Consequentemente, várias tarefas são desenvolvidas para preservar o meio ambiente e buscar a sustentabilidade.

Palavras-chave: competências ambientais, crise ambiental, currículo, universidades. Fecha Recepción: Julio 2019 Fecha Aceptación: Octubre 2019 


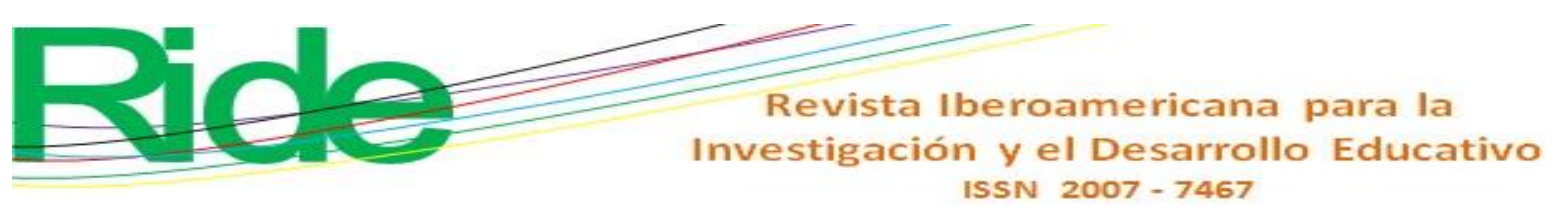

de Guerrero (UAGro), con sede en Acapulco, México; el programa de la Facultad de Educación de la Universidad Metropolitana Nelson Mandela (UMNM) en Puerto Elizabeth, Sudáfrica, y los programas de la Licenciatura en Biología y Arquitectura y Urbanismo de la Universidad Regional de Blumenau (FURB), en Brasil.

Es una investigación mixta con enfoque comparativo y descriptivo. Se realizó en el periodo 2015-2017. Entre los años 2015-2016 se trabajó con los programas educativos de la UAGro. En el 2015, a finales, con el programa de Sudáfrica. Durante el mes de noviembre de 2017 se trabajó con los programas de la FURB. Se aplicó una encuesta a profesores y a estudiantes en dichos objetos de estudio; una entrevista a profesores informantes claves del currículo en los programas de México y Sudáfrica, y cuestionarios de opinión también a informantes clave del currículo en los programas de la universidad de Brasil. Se revisaron algunos documentos oficiales como reglamentos, planes de estudio y modelo educativo. Estos instrumentos y técnicas tuvieron como base los elementos medio ambiente-problemática ambiental, EA y currículo, competencias ambientales, documentos oficiales que establecen la incorporación de EA. En sí, se enfocaron en comprender la percepción de la problemática ambiental, en la relación que existe entre la EA y el currículo, las competencias ambientales que promueven docentes y forman estudiantes, la revisión de reglamentos que establecen la integración de este enfoque en el plan de estudio, con la finalidad de cumplir los propósitos del estudio.

El resultado de la encuesta evidenció que estudiantes y profesores de los programas educativos de México cuentan con menor grado de percepción de la problemática ambiental en comparación con los profesores de los programas de las otras dos universidades. Con respecto a la relación de la EA y el currículo, estudiantes y profesores de Sudáfrica señalaron que el currículo no se vincula con esta, los de México indicaron que muy poco y los de Brasil que sí está integrada pero su inclusión está en proceso. Mientras que, respecto a las competencias ambientales, los estudiantes y profesores del programa de Sudáfrica no las promueven; en cambio en los programas de México y Brasil mencionan que sí, aunque falta fortalecerlas. De la información proporcionada por informantes claves se dedujo que dos programas de la universidad de México y los dos de Brasil contemplan de alguna forma la EA. No obstante, en aquellos es en menor proporción y de manera disciplinar; y en estos últimos es en mayor proporción: su integración es de manera transversal y está en proceso. En el programa de Sudáfrica, la EA no figura. En cuanto a los documentos oficiales, los programas de México y Brasil cuentan con un sustento que respalda la integración de la EA. En Sudáfrica, por su parte, no se cuenta con un documento oficial. La problemática ambiental va en aumento e impacta en diferentes aspectos. Actualmente no solo es 


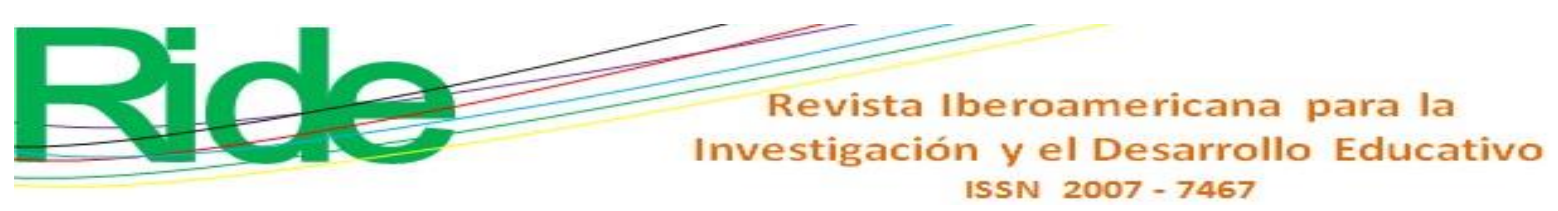

cuestión de educar para la "naturaleza", es decir, no confundamos la EA con biología o ecología, pues va más allá, tiene que ver con naturaleza, sociedad y economía, entre otras. Las universidades en todo el mundo deben adquirir la responsabilidad de integrar esta educación en sus currículos, en sus diferentes programas educativos. De esta forma, se atendería a las indicaciones que organismos internacionales sugieren para contribuir en un mundo sustentable.

\section{Problemáticas ambientales, socioambientales y su relación con el contexto educativo}

En relación con los problemas ambientales y socioambientales, algunos autores plantean distinciones, y hace referencia, en el primer caso, a las cuestiones relativas al desgaste que están sufriendo los recursos naturales; en el segundo caso, a la incorporación de estas mismas cuestiones a las comunidades directamente afectadas por los impactos derivados de un determinado proyecto (Moreno- Crespo y Moreno Fernández, 2015, p. 76). Los problemas propiamente ecológicos impactan en lo social. Ejemplo de ello es la tala de árboles, la contaminación del agua, las altas temperaturas, que se asocian con problemas de salud, escasez de agua en las comunidades, el bajo rendimiento de cultivos, las tierras fértiles se escasean entre otros. En muchos casos, esto tiene su origen en las actividades humanas que se llevan a cabo de manera irracional.

El Programa de las Naciones Unidas para el Medio Ambiente [Pnuma] (2019), en su Resumen para responsables de formular políticas, expuso que la problemática socioambiental que se visibiliza en el mundo proviene de las emisiones antropógenas que siguen alterando la composición de la atmósfera, lo que da lugar a la contaminación del aire, el cambio climático. La contaminación del aire es el principal factor ambiental que contribuye a la carga mundial de morbilidad, y ocasiona entre 6 y 7 millones de muertes prematuras. Se está desencadenando un importante proceso de extinción de especies, el calentamiento y aumento de los océanos, el crecimiento demográfico, la urbanización, la contaminación del agua, la degradación y desertificación de las tierras. La temática del deterioro ambiental es una de las problemáticas que más atención centra entre las inquietudes que afectan a la humanidad en la actualidad. Desde las conversaciones habituales del ciudadano común hasta los debates entre versados expertos en este campo del conocimiento, la situación del ambiente es objeto de diversos puntos de vista, enfoques y marcos teóricos en muchas regiones del planeta (Santiago, 2009). "Numerosos autores destacan la importancia de los problemas sociocientíficos como contexto de aprendizaje (Oulton, Dillon y Grace, 2004; Sadler, 2009; Zeidler et al., 2002), entre los cuales se incluyen problemáticas reales, 


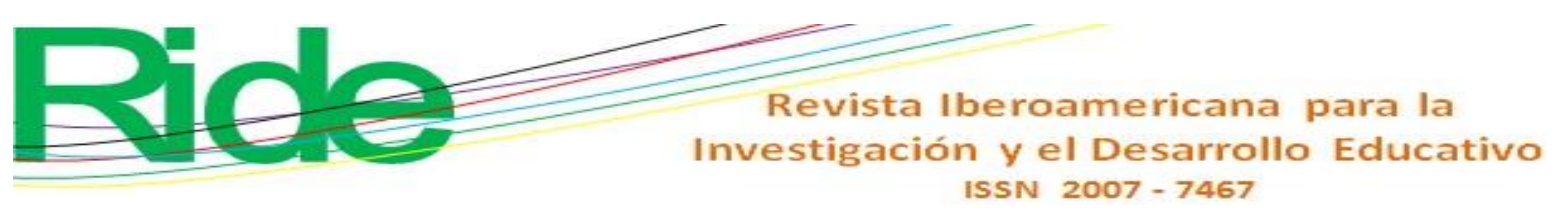

en muchos casos cercanas, complejas y controvertidas dada su afectación o incidencia local-global sin soluciones únicas" (Moreno-Crespo y Moreno-Fernández, 2015, p. 76). Al tratar los problemas socioambientales en los centros educativos, encontramos que terminan por "invisibilizarse" en el currículo escolar, pasándose por alto o incluso desapareciendo por completo a pesar de estar presentes en la realidad cotidiana de nuestros.

\section{Integración de la educación en el currículo universitario}

Al respecto, Nieto y Buendía (2008) manifiestan que el proyecto educativo no está aislado de la realidad en que busca incidir. Este debe plantear una forma de interpretar la relación entre educación y realidad social. La contextualización de currículo se plantea aquí como la acción de situar a este en un espacio de intervención multicultural socialmente complejo (Mallarino, 2007). "Plantearse esta temática de la contextualización curricular exige, en primer lugar, un esfuerzo de clarificación conceptual porque la idea de vincular currículo, escuela y contextos genera una tupida red de lazos semánticos y significados no siempre coincidentes" (Zabalza, 2012, p. 8). La emergencia y manifestaciones de una crisis ambiental obligan a la sociedad a analizar el origen del deterioro ambiental y proponer acciones que permitan frenar el deterioro ambiental. La ambientalización curricular se posiciona como la respuesta de las instituciones educativas ante el compromiso de la sustentabilidad. Las estrategias de ambientalización universitaria se establecen básicamente en tres ámbitos: 1) ambientalización curricular, 2) gestión ambiental sostenible y 3) educación y participación ambiental. La ambientalización curricular consiste en introducir contenidos ambientales en el currículo (Herrera, 2013).

\section{Educación ambiental como tema disciplinar y transversal}

La EA como tema disciplinar y transversal considera integrar contenidos ambientales. Sin embargo, para que sea considerada como un tema transversal en un currículo debe estar presente en todas las actividades educativas que realice una institución e involucrar a todos los integrantes de la misma (Rengifo, Quitiaquez y Mora, 2012). Así, pues, debe estar presente en todas las unidades de aprendizaje y reflejarse en el proceso de enseñanza-aprendizaje y no integrarla solo como una asignatura con temas ambientales propias de una disciplina. No debe ser considerada como "unidades didácticas aisladas, sino como ejes claros de objetivos, contenidos y principios de procedimiento que han de dar coherencia y solidez a las materias y salvaguardar sus interconexiones en la medida de lo posible" (Conde, 2004, p. 59). 


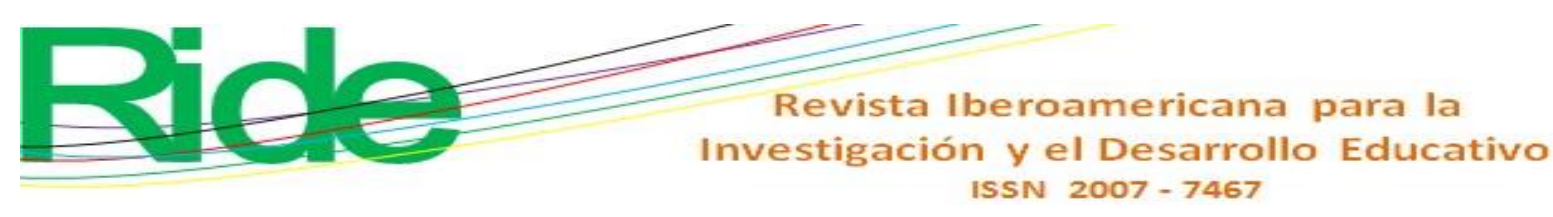

Tabla 1. Población y muestra seleccionada de las tres universidades

\begin{tabular}{|c|c|c|c|c|c|c|}
\hline \multicolumn{2}{|c|}{ Población } & $\begin{array}{c}\text { Programa } \\
\text { s } \\
\text { selecciona } \\
\text { dos }\end{array}$ & $\begin{array}{c}\text { Población de } \\
\text { los } \\
\text { programas } \\
\text { seleccionado } \\
\text { s para la } \\
\text { investigación } \\
\text { y edad }\end{array}$ & Muestra & $\begin{array}{c}\text { Enfoque } \\
\text { de } \\
\text { selección } \\
\text { de la } \\
\text { muestra } \\
\text { y edad } \\
\text { de los } \\
\text { particip } \\
\text { antes }\end{array}$ & $\begin{array}{c}\text { Otros } \\
\text { sujetos } \\
\text { consider } \\
\text { ados en } \\
\text { el } \\
\text { estudio }\end{array}$ \\
\hline UAGro & \begin{tabular}{|l} 
*Profesore \\
s: $1162:$ \\
383 \\
mujeres y \\
779 \\
hombres \\
\\
Estudiante \\
s: 83770 \\
\\
39302 \\
hombres y \\
43468 \\
mujeres
\end{tabular} & $\begin{array}{l}\begin{array}{l}\text { Facultad } \\
\text { de } \\
\text { Sociología }\end{array} \\
\text { Facultad } \\
\text { de } \\
\text { Economía } \\
\\
\text { Facultad } \\
\text { de } \\
\text { Derecho }\end{array}$ & $\begin{array}{l}15 \text { profesores } \\
\text { y } 287 \\
\text { estudiantes } \\
\text { de sociología } \\
15 \text { profesores } \\
\text { y } 259 \\
\text { estudiantes } \\
\text { de economía } \\
\\
48 \text { profesores } \\
\text { y } 2044 \\
\text { estudiantes } \\
\text { de derecho }\end{array}$ & $\begin{array}{l}4 \text { profesores } \\
\text { y } 5 \\
\text { estudiantes } \\
11 \\
\text { profesores y } \\
91 \\
\text { estudiantes } \\
44 \\
\text { profesores y } \\
318 \\
\text { estudiantes } \\
\text { de } \\
\text { diferentes } \\
\text { grados de la } \\
\text { licenciatura. }\end{array}$ & $\begin{array}{l}\text { Cualitati } \\
\text { vo } \\
\text { La edad } \\
\text { de los } \\
\text { estudiant } \\
\text { es oscila } \\
\text { entre 18- } \\
23 \text { años. }\end{array}$ & $\begin{array}{l}\text { Profesor } \\
\text { es } \\
\text { informan } \\
\text { te clave } \\
\text { del } \\
\text { program } \\
\text { a } \\
\text { educativ } \\
\text { o }\end{array}$ \\
\hline NMMU & $\begin{array}{l}\text { **Profesor } \\
\text { es } \\
\text { permanent } \\
\text { es } 223 \text { y } \\
\text { temporales } \\
1029 \\
\text { Estudiante } \\
\text { s: } 26347 \\
39302 \\
\text { hombres y } \\
43468 \\
\text { mujeres }\end{array}$ & $\begin{array}{l}\text { Facultad } \\
\text { de } \\
\text { Educación }\end{array}$ & $\begin{array}{l}85 \\
\text { profesores: } \\
44 \quad \text { son } \\
\text { permanentes } \\
\text { y } 41 \text { son } \\
\text { temporales } \\
1779 \\
\text { estudiantes: } \\
548 \text { son } \\
\text { hombres y } \\
1231 \text { son } \\
\text { mujeres }\end{array}$ & $\begin{array}{l}10 \\
\text { profesores: } \\
5 \text { hombres y } \\
5 \text { mujeres. } \\
46 \\
\text { estudiantes: } \\
12 \text { hombres } \\
\text { y } 34 \\
\text { mujeres. De } \\
\text { diferentes } \\
\text { grados de la } \\
\text { licenciatura }\end{array}$ & $\begin{array}{l}\text { Cualitati } \\
\text { vo } \\
\text { La edad } \\
\text { de los } \\
\text { estudiant } \\
\text { es oscila } \\
\text { entre 18- } \\
23 \text { años. }\end{array}$ & $\begin{array}{l}\text { Profesor } \\
\text { es } \\
\text { informan } \\
\text { te clave } \\
\text { del } \\
\text { program } \\
\text { a } \\
\text { educativ } \\
\text { o }\end{array}$ \\
\hline FURB & $\begin{array}{l}* * \text { Profes } \\
\text { ores entre } \\
862 \\
\text { hombres y } \\
\text { mujeres }\end{array}$ & $\begin{array}{l}\text { Cursos de } \\
\text { Ciencias } \\
\text { Biológicas }\end{array}$ & $\begin{array}{l}38 \text { profesores } \\
\text { y } 86 \\
\text { estudiantes }\end{array}$ & $\begin{array}{l}10 \\
\text { Profesores } \\
\text { (6) hombres } \\
\text { (4) mujeres. } \\
37 \\
\text { Estudiantes } \\
\text { (17) }\end{array}$ & $\begin{array}{l}\text { Cualitati } \\
\text { vo } \\
\text { La edad } \\
\text { de los } \\
\text { estudiant } \\
\text { es oscila }\end{array}$ & $\begin{array}{l}\text { Conversa } \\
\text { ciones } \\
\text { con } \\
\text { informan } \\
\text { tes } \\
\text { claves } \\
\text { del }\end{array}$ \\
\hline
\end{tabular}


Revista Iberoamericana para la
Investigación y el Desarrollo Educativo

ISSN $2007-7467$

\begin{tabular}{|c|c|c|c|c|c|c|}
\hline & $\begin{array}{l}\text { Estudiante } \\
\text { s (no se } \\
\text { cuenta con } \\
\text { el número } \\
\text { total de la } \\
\text { población } \\
\text { estudiantil } \\
\text { ) }\end{array}$ & $\begin{array}{l}\text { Curso de } \\
\text { Arquitectu } \\
\text { ra y } \\
\text { Urbanism } \\
\text { o }\end{array}$ & $\begin{array}{l}41 \\
\text { profesores, } \\
\text { no hay datos } \\
\text { de la } \\
\text { matrícula de } \\
\text { estudiantes }\end{array}$ & $\begin{array}{l}\text { hombres, } \\
(20) \\
\text { mujeres. } \\
\text { Profesores: } \\
\text { 11: } 7 \\
\text { hombres y } 4 \\
\text { mujeres } \\
\text { Estudiantes: } \\
\text { 36: } 2 \\
\text { hombres y } \\
34 \text { mujeres } \\
\text { Diferentes } \\
\text { grados }\end{array}$ & $\begin{array}{l}\text { entre } 18- \\
23 \text { años }\end{array}$ & $\begin{array}{l}\text { program } \\
\text { a }\end{array}$ \\
\hline
\end{tabular}

** Dr. Logamurthie Athiemoolam, profesor de la Facultad de Educación de la NMMU.

*** Profesor de la FURB.

Fuente: Elaboración propia con base en Anuario Estadístico 2013-2014 y Anuario Estadístico

2014-2015 de la UAGro (2014a, 2015)

\section{Técnicas e instrumentos empleados}

Se aplicaron técnicas e instrumentos con un enfoque cuantitativo y cualitativo para los diferentes programas de las tres universidades. Para los programas educativos de sociología, economía y derecho pertenecientes a la UAGro se implementó la técnica cuantitativa a través de la encuesta a estudiantes que contempló las variables: percepción del problema, componentes del elemento ambiental, currículo y competencias. El objetivo del instrumento fue identificar la dimensión ambiental en los programas educativos. También se aplicó una encuesta a docentes con las variables: percepción del problema, componentes del elemento ambiental, currículo y competencias. El objetivo de este fue identificar la dimensión ambiental en los programas educativos.

Otra técnica cualitativa considerada fue la entrevista con informantes claves del currículo, con las variables: componentes del medio ambiente, competencias y currículo; con el objetivo de conocer la opinión de los directivos de los programas educativos sobre la integración de la dimensión ambiental.

Se analizaron documentos oficiales como el Modelo educativo de la UAGro (2004) con el objetivo de realizar un análisis sobre la incorporación del elemento medio ambiente en programas de estudio de la universidad.

Para la Facultad de Educación de la NMMU, en Sudáfrica, se realizó una encuesta a estudiantes con las variables: percepción del problema, componentes del elemento ambiental, 


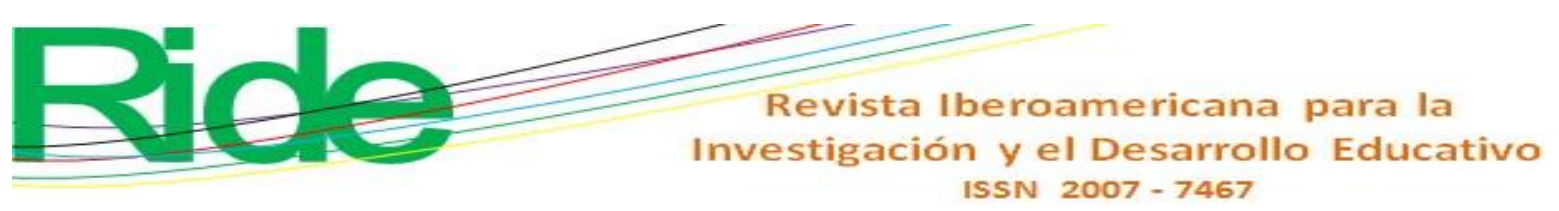

currículo y competencias, con el objetivo de identificar la dimensión ambiental en los programas educativos. Asimismo, se aplicó una encuesta a profesores con las variables: problemática ambiental, el currículo y medio ambiente, el papel del profesor, del estudiante y las competencias ambientales. El objetivo del instrumento fue identificar la dimensión ambiental en el programa educativo.

Y también se realizó una entrevista con informantes claves del currículo. Para esta se consideraron las variables: componentes del medio ambiente, competencias y currículo; con el objetivo de conocer la opinión de los directivos de los programas educativos sobre la integración de la dimensión ambiental. En este caso no se analizó el documento oficial de la universidad.

En la FURB se trabajó con dos programas, el de Ciencias Biológicas y de Arquitectura y Urbanismo. Para el primero se consideró un cuestionario de opinión a profesores y estudiantes con el objetivo de comprender la incorporación de la EA en el plan de estudio; para el segundo curso se aplicó un cuestionario de opinión a profesores y estudiantes. También se consideran técnicas de corte cualitativo: conversación con informantes claves de la especialidad de Biología, con el objetivo de analizar la dimensión ambiental el currículo. En el curso de Arquitectura y Urbanismo también se llevaron a cabo conversaciones con profesores.

Se analizó el documento oficial "Resolución N N . 2, 15 de junio de 2012. Directrices Curriculares Nacionales para la Educación Ambiental del Ministerio de Educación, el Consejo Nacional de Educación de Brasil y Resolución 053/2014/Rectoría, Universidade Regional de Blumenau", que establece las directrices nacionales para la EA en dicha institución, así como los planes de estudio de las Licenciaturas en Biología y Arquitectura y Urbanismo. Esto con el objetivo de realizar un análisis sobre la incorporación del elemento medio ambiente en programas de estudio de la universidad.

\section{Resultados}

Las técnicas e instrumentos aplicados en los programas educativos universitarios para esta investigación permitieron comprender en qué medida la EA figura en el currículo. 


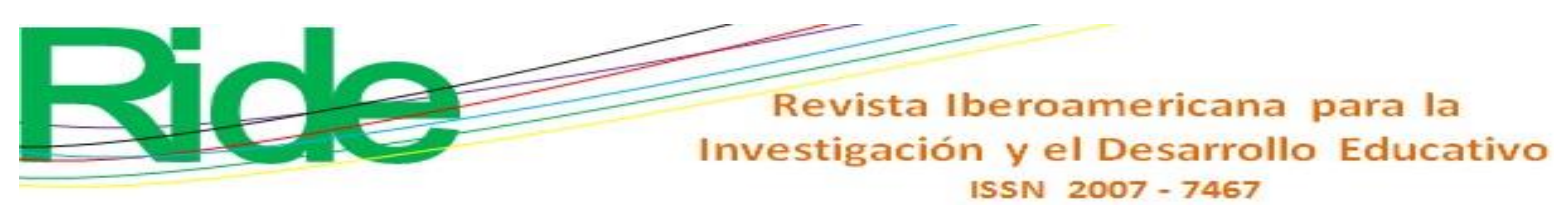

Programas educativos en la UAGro

\section{Resultados de la encuesta a estudiantes y docentes}

Se aplicó una encuesta a estudiantes y docentes, la cual se enmarcó en las variables percepción del problema, componentes del elemento ambiental, currículo y competencias, y se relacionó con los elementos del medio ambiente (agua, aire, suelo y energía). Las preguntas consideraron los siguientes parámetros: "Deficiente", "Apenas aceptable”, "Bien”, "Muy bien”, "Excelente" y "No aplica". Los resultados pudieron agruparse en "Deficiente", "Apenas aceptable", "Bien", "Muy bien” y "Excelente". En la tabla 2 se presentan los resultados obtenidos en los tres programas educativos objeto de estudio correspondientes a la UAGro.

Tabla 2. Resultados de los programas de sociología, economía y derecho

\begin{tabular}{|c|c|c|c|c|}
\hline Variables & $\begin{array}{l}\text { Escuela de } \\
\text { Sociología }\end{array}$ & $\begin{array}{l}\text { Escuela de } \\
\text { Economía }\end{array}$ & $\begin{array}{ll}\text { Escuela de } \\
\text { Derecho }\end{array}$ & Observación \\
\hline $\begin{array}{l}\text { Percepción del } \\
\text { problema }\end{array}$ & Deficiente & Bien & $\begin{array}{l}\text { Apenas } \\
\text { aceptable }\end{array}$ & $\begin{array}{l}\text { Apenas conciben el } \\
\text { problema ambiental. }\end{array}$ \\
\hline Currículo & Deficiente & Bien & $\begin{array}{l}\text { Apenas } \\
\text { aceptable }\end{array}$ & $\begin{array}{l}\text { Apenas contempla el } \\
\text { currículo el elemento } \\
\text { medio ambiente y por ende } \\
\text { la EA. Los programas de } \\
\text { unidades de aprendizaje y } \\
\text { las actividades educativas } \\
\text { en su mayoría no integran } \\
\text { la EA. }\end{array}$ \\
\hline $\begin{array}{l}\text { Competencias en } \\
\text { los alumnos }\end{array}$ & $\begin{array}{l}\text { Apenas } \\
\text { aceptable }\end{array}$ & Bien & Deficiente & $\begin{array}{l}\text { Apenas desarrollan } \\
\text { competencias ambientales. } \\
\text { Los profesores no } \\
\text { implementan estrategias de } \\
\text { la EA. }\end{array}$ \\
\hline
\end{tabular}

Fuente: Elaboración propia

En la tabla 3 se presentan los resultados de la encuesta aplicada a profesores de los tres programas de estudio de la UAGro. 


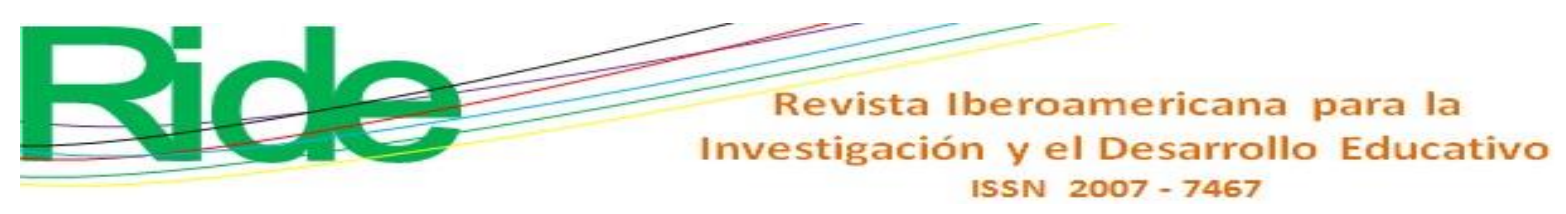

Tabla 3. Resultados de los programas de sociología, economía y derecho

\begin{tabular}{|l|l|l|l|l|}
\hline Variables & $\begin{array}{l}\text { Facultad } \\
\text { de } \\
\text { Sociología }\end{array}$ & $\begin{array}{l}\text { Facultad de } \\
\text { Economía }\end{array}$ & $\begin{array}{l}\text { Facultad } \\
\text { de Derecho }\end{array}$ & Observación \\
\hline $\begin{array}{l}\text { Percepción } \\
\text { del } \\
\text { problema }\end{array}$ & Deficiente & $\begin{array}{l}\text { Apenas } \\
\text { aceptable }\end{array}$ & $\begin{array}{l}\text { Apenas } \\
\text { aceptable }\end{array}$ & $\begin{array}{l}\text { Apenas comprenden la problemática } \\
\text { ambiental. }\end{array}$ \\
\hline Currículo & $\begin{array}{l}\text { Apenas } \\
\text { aceptable }\end{array}$ & Deficiente & $\begin{array}{l}\text { Apenas } \\
\text { aceptable }\end{array}$ & $\begin{array}{l}\text { Apenas contempla el elemento } \\
\text { ambiental por ende la EA en el } \\
\text { currículo. Los planes de estudio, los } \\
\text { programas de unidades de } \\
\text { aprendizaje y la secuencia didáctica } \\
\text { están escasamente vinculados con la } \\
\text { EA. }\end{array}$ \\
\hline $\begin{array}{l}\text { Competenci } \\
\text { as } \\
\text { ambientales } \\
\text { de } \\
\text { docentes }\end{array}$ & $\begin{array}{l}\text { Apenas } \\
\text { aceptable }\end{array}$ & Bien & Deficiente & $\begin{array}{l}\text { Se concibe actualizar a los docentes } \\
\text { en aspectos ambientales. Muchos } \\
\text { docentes no cuentan con formación } \\
\text { en EA, por lo tanto, difícilmente } \\
\text { implementan estrategias didácticas } \\
\text { de esta especialidad en el proceso de } \\
\text { aprendizaje. Los estudiantes no } \\
\text { promueven competencias } \\
\text { ambientales. }\end{array}$ \\
\hline
\end{tabular}

Fuente: Elaboración propia

\section{Entrevista con informantes claves de los programas de la UAGro}

Se realizó una entrevista al personal directivo de la Facultad de Sociología (director), de Derecho (subdirector) y de Economía (subdirector), dependientes de la UAGro, ubicada en Acapulco, Guerrero, México. Estas se desarrollaron durante el periodo de marzo a octubre de 2015. El objetivo fue conocer si el elemento medio ambiente está incorporado en estos planes de estudios, planteado como una hipótesis en cualquier momento. En la tabla 4 se muestra la opinión del personal directivo de los tres programas. 


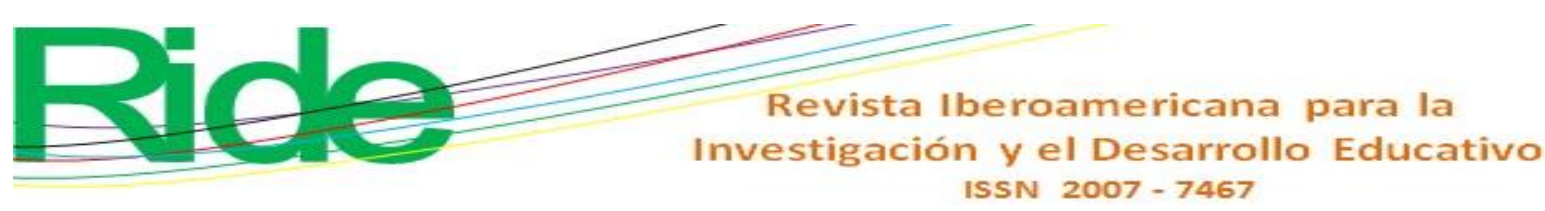

ejes transversales y lo considera como tema transversal, al igual que otros, y con ello también manifiesta que estos son "instrumentos globalizantes de carácter interdisciplinario que recorren la totalidad de un currículo; son condiciones favorables para proporcionar a los alumnos una mayor formación en aspectos sociales, ambientales o de salud" Botero (citado en UAGro, 2013, p.102). Por otro lado, un principio rector de este modelo es la sustentabilidad (UAGro, 2013). Estas aseveraciones sugieren que en todos los niveles que imparte la institución en cuestión se incorpore el elemento medio ambiente, con un enfoque transversal para que se coadyuve al desarrollo sustentable.

\section{Resultados del programa educativo en la Facultad de Educación de la UMNM Resultados de la encuesta a estudiantes y docentes}

La encuesta que se aplicó a estudiantes y docentes y se enmarcó en las siguientes variables: percepción del problema ambiental, currículo y medio ambiente, competencias ambientales del profesor y estudiante; igualmente se relacionó con los elementos del medio ambiente (agua, aire, suelo y energía). Las preguntas consideraron los parámetros "Deficiente", "Apenas aceptable", "Bien", "Muy bien", "Excelente" y "No aplica”. Los resultados pudieron agruparse en porcentajes. Ambas encuestas tenían como objetivo identificar la dimensión ambiental en el programa educativo seleccionado en la UMNM. En la tabla 5 se presentan los resultados obtenidos de la encuesta aplicada a estudiantes. El instrumento se aplicó a 46 estudiantes (12 hombres, 34 mujeres). La tabla 6, por su parte, corresponde a los resultados de los profesores; datos proporcionados por 10 docentes (cinco hombres y cinco mujeres). 


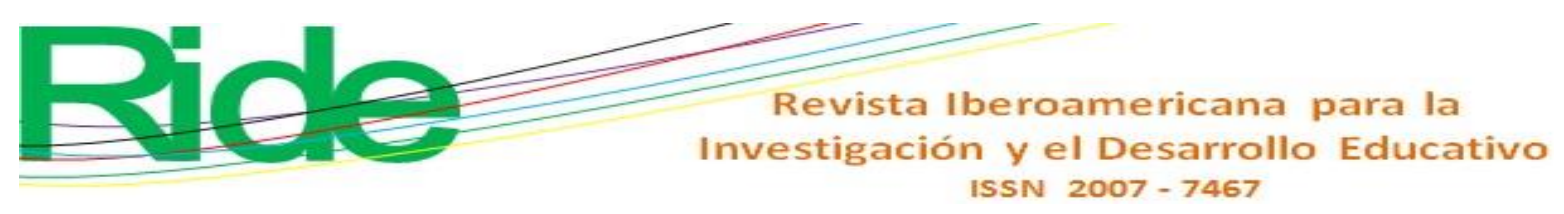

Tabla 5. Resultados de la encuesta a estudiantes UMNM

\begin{tabular}{|c|c|c|c|c|c|c|c|c|c|c|c|c|}
\hline \multirow[t]{2}{*}{ Dimensiones } & \multirow{2}{*}{$\begin{array}{l}\text { Ítems } \\
\text { Las preguntas } \\
\text { realizadas } \\
\text { tuvieron } \\
\text { relación con las } \\
\text { dimensiones } \\
\text { establecidas. }\end{array}$} & \multicolumn{11}{|c|}{ Porcentaje alcanzado } \\
\hline & & $\begin{array}{l}0 \\
\%\end{array}$ & $\begin{array}{l}1 \\
0 \\
\%\end{array}$ & $\begin{array}{l}2 \\
0 \\
\%\end{array}$ & $\begin{array}{l}3 \\
0 \\
\%\end{array}$ & $\begin{array}{l}4 \\
0 \\
\%\end{array}$ & $\begin{array}{l}5 \\
0 \\
\%\end{array}$ & $\begin{array}{l}6 \\
0 \\
\%\end{array}$ & \begin{tabular}{|l|}
7 \\
0 \\
$\%$
\end{tabular} & $\begin{array}{l}8 \\
0 \\
\%\end{array}$ & $\begin{array}{l}9 \\
0 \\
\%\end{array}$ & $\begin{array}{l}1 \\
0 \\
0 \\
\%\end{array}$ \\
\hline $\begin{array}{l}\text { 1. Percepción de la } \\
\text { problemática } \\
\text { ambiental }\end{array}$ & $\begin{array}{l}\text { Perciben la } \\
\text { crisis ambiental } \\
\text { en un contexto } \\
\text { general y local, } \\
\text { sin embargo, } \\
\text { esta percepción } \\
\text { no se originó en } \\
\text { el desarrollo } \\
\text { del currículo. }\end{array}$ & & & & & & & & & & $\mathbf{X}$ & \\
\hline $\begin{array}{l}\text { 2. La integración del } \\
\text { elemento ambiental } \\
\text { en el currículo }\end{array}$ & $\begin{array}{l}\text { El currículo de } \\
\text { la Facultad de } \\
\text { Educación de la } \\
\text { NMMU integra } \\
\text { la EA. }\end{array}$ & $\mathbf{X}$ & & & & & & & & & & \\
\hline \multirow[t]{2}{*}{$\begin{array}{l}\text { 3. La comprensión de } \\
\text { saberes ambientales } \\
\text { en estudiantes }\end{array}$} & $\begin{array}{l}\text { Los estudiantes } \\
\text { desarrollan } \\
\text { competencias } \\
\text { ambientales. }\end{array}$ & $\mathbf{X}$ & & & & & & & & & & \\
\hline & $\begin{array}{l}\text { Competencias } \\
\text { ambientales de } \\
\text { docentes. }\end{array}$ & $\mathbf{X}$ & & & & & & & & & & \\
\hline
\end{tabular}

Fuente: Elaboración propia 


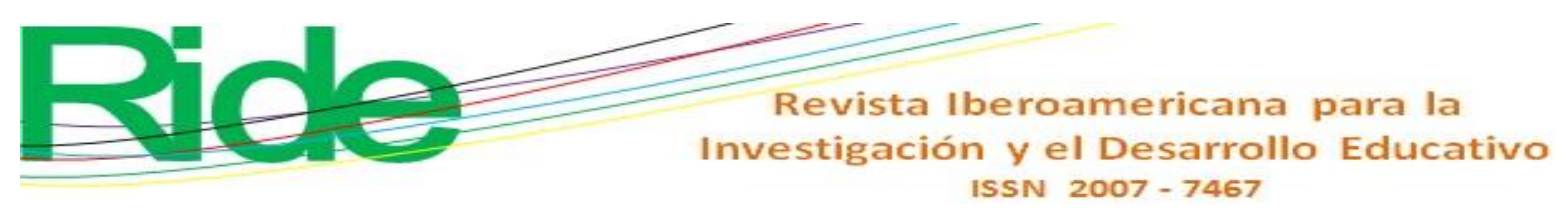

Tabla 6. Resultados de la encuesta a profesores UMNM

\begin{tabular}{|c|c|c|c|c|c|c|c|c|c|c|c|c|}
\hline \multirow[t]{2}{*}{ Dimensiones } & \multirow{2}{*}{$\begin{array}{l}\text { Items } \\
\text { Las preguntas realizadas } \\
\text { tuvieron relación con las } \\
\text { dimensiones } \\
\text { establecidas. }\end{array}$} & \multicolumn{11}{|c|}{ Porcentaje alcanzado } \\
\hline & & 0 & 10 & 20 & 30 & 40 & 50 & 60 & 70 & 80 & 90 & 100 \\
\hline $\begin{array}{l}\text { 1. Percepción de } \\
\text { la problemática } \\
\text { ambiental }\end{array}$ & $\begin{array}{l}\text { Comprenden la } \\
\text { problemática ambiental } \\
\text { y están de acuerdo con } \\
\text { que el currículo integre } \\
\text { esta dimensión. }\end{array}$ & & & & & & & & & & & $\mathbf{X}$ \\
\hline $\begin{array}{l}\text { 2. La integración } \\
\text { del elemento } \\
\text { ambiental en el } \\
\text { currículo }\end{array}$ & $\begin{array}{l}\text { El currículo de la } \\
\text { Facultad de Educación } \\
\text { de la NMMU integra la } \\
\text { EA. }\end{array}$ & $\mathbf{X}$ & & & & & & & & & & \\
\hline \multirow{2}{*}{$\begin{array}{l}\text { 3. La comprensión } \\
\text { de saberes } \\
\text { ambientales en } \\
\text { estudiantes }\end{array}$} & $\begin{array}{l}\text { Competencias } \\
\text { ambientales } \\
\text { estudiantes. }\end{array}$ & $\mathbf{X}$ & & & & & & & & & & \\
\hline & $\begin{array}{l}\text { Competencias } \\
\text { ambientales } \\
\text { docentes. }\end{array}$ & $\mathbf{X}$ & & & & & & & & & & \\
\hline
\end{tabular}

Fuente: Elaboración propia

\section{Resultados de la entrevista con informante clave del currículo}

Se llevó a cabo una entrevista con un profesor informante clave de la Facultad de Educación en la UMNM de Sudáfrica. Comentó que en el currículo no se establece la dimensión ambiental.

\section{Programas Educativos en la FURB}

\section{Resultados del cuestionario de opinión a profesores y estudiantes de la Licenciatura en Biología y de Arquitectura y Urbanismo}

El cuestionario de opinión contenía 32 enunciados agrupados en cinco dimensiones: 1) Conocimiento general de la problemática ambiental, 2) Percepción ambiental en el currículo, 3) Implementación didáctica pedagógica ambiental, 4) Competencias ambientales de profesores y 5) Competencias ambientales de estudiantes. Se elaboró para docentes y estudiantes. Las respuestas se respondieron con Totalmente de acuerdo (5), De acuerdo (4), Moderadamente de acuerdo (3), En desacuerdo (2) y Totalmente en desacuerdo (1). Las respuestas obtenidas se organizaron por dimensiones y se presentan en escalas de 0 a $100 \%$. En la tabla 7 se describen los resultados de docentes y estudiantes de ambos programas; figuran de la siguiente manera: Estudiantes de la Lic. 


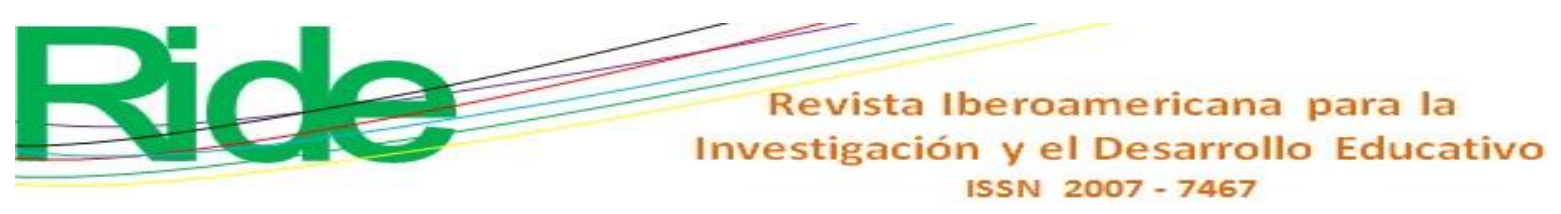

en Biología (ECB) y profesores de este mismo programa (PCB). Estudiantes de la Lic. de Arquitectura y Urbanismo (EAU) y los profesores (PAU).

Tabla 7. Resultados de los programas de Biología y Arquitectura y Urbanismo

\begin{tabular}{|c|c|c|c|c|c|c|c|c|c|c|c|}
\hline Dimensiones & 0 & 10 & 20 & 30 & 40 & 50 & 60 & 70 & 80 & 90 & 100 \\
\hline $\begin{array}{l}\text { Conocimiento } \\
\text { general de la } \\
\text { problemática } \\
\text { ambiental }\end{array}$ & & & & & & & & & & $\begin{array}{l}\text { ECB } \\
\text { EAU }\end{array}$ & $\begin{array}{l}\text { PCB } \\
\text { PAU }\end{array}$ \\
\hline $\begin{array}{l}\text { Percepción } \\
\text { ambiental en el } \\
\text { currículo }\end{array}$ & & & & & & & & & & $\begin{array}{l}\text { ECB } \\
\text { EAU }\end{array}$ & $\begin{array}{l}\text { PCB } \\
\text { PAU }\end{array}$ \\
\hline $\begin{array}{l}\text { Implementació } \\
\text { n didáctico- } \\
\text { pedagógica } \\
\text { ambiental }\end{array}$ & & & & & & & & EAU & ECB & $\begin{array}{l}\text { PCB } \\
\text { PAU }\end{array}$ & \\
\hline $\begin{array}{l}\text { Competencias } \\
\text { ambientales } \\
\text { docentes }\end{array}$ & & & & & & & & & $\begin{array}{l}\text { ECB } \\
\text { EA } \\
\text { U }\end{array}$ & $\begin{array}{l}\text { PCB } \\
\text { PAU }\end{array}$ & \\
\hline $\begin{array}{l}\text { Competencias } \\
\text { ambientales de } \\
\text { estudiantes }\end{array}$ & & & & & & & & $\begin{array}{l}\text { ECB } \\
\text { EAU } \\
\text { PCB } \\
\text { PAU }\end{array}$ & & & \\
\hline
\end{tabular}

Fuente: Elaboración propia

\section{Conversación con informantes claves de Biología y Arquitectura y Urbanismo}

De acuerdo con la conversación con informantes claves de la Lic. en Biología, se percibió que la EA se integra como una disciplina y está en proceso su transversalización. La conversación con los profesores de Biología y también los de Arquitectura y Urbanismo arrojó que la EA sí se hace presente debido a que varias disciplinas se relacionan con el medio en el momento en que se desarrollan proyectos. 


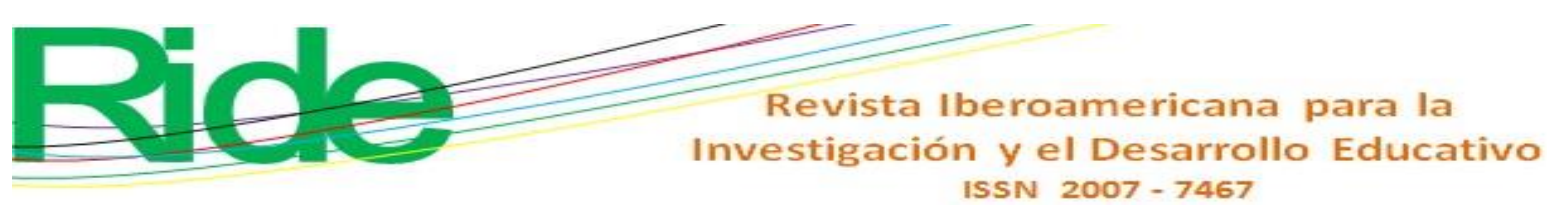

\section{Resultado de los documentos oficiales para identificar la dimensión ambiental en los programas estudiados}

La "Resolución No. 2, 15 de junio de 2012. Directrices Curriculares Nacionales para la Educación Ambiental" es el sustento legal para que toda institución que imparta educación en Brasil implemente curricularmente la EA. Con base en dicho documento y de acuerdo a un análisis realizado, se afirma entonces que el currículo de la Licenciatura en Ciencias Biológicas y el de Arquitectura y Urbanismo de la FURB se relaciona con lo que se establece en dicha resolución, y se destaca el elemento medio ambiente con enfoque sustentable en sus elementos curriculares de los programas.

La "Resolución 053/2014/Rectoría, FURB" coincide con lo estipulado, que establece las directrices curriculares nacionales para la educación ambiental del Ministerio de Educación de Brasil.

De acuerdo a un análisis realizado en los elementos curriculares de la Lic. en Biología y Arquitectura y Urbanismo de la FURB, se evidenció que el elemento medio ambiente con enfoque sustentable se hace presente en ambos programas, aunque aún está en proceso su transversalización. Ello lleva a comprender que hay una relación entre el currículo y los documentos normativos.

\section{Discusión}

Se realizó un análisis comparativo de los programas de las tres universidades para identificar| la incorporación de la EA por parte de estudiantes y docentes. A partir de ello se evidenció que los estudiantes de los tres programas de México perciben en bajo nivel la problemática que se vive en el mundo, en su contexto social y educativo. Mientras que en los programas de las otras dos universidades su percepción es mayor. Respecto a los docentes, es el mismo caso: los de México siguen estando en niveles por debajo de los programas con los que se le compara. En lo que concierne a la relación que existe entre la EA y el currículo, los estudiantes del programa de la universidad de Sudáfrica señalan que el currículo no se vincula con la EA. En el caso de los estudiantes de México, estos establecieron que esta educación sí figura, pero no es suficiente. Y los estudiantes y docentes de los programas de Brasil en ambos programas indicaron que este sí se desarrolla. 


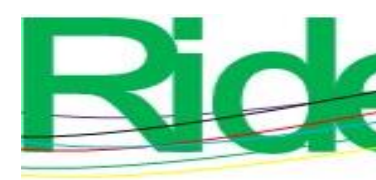

\section{Revista Iberoamericana para la Investigación y el Desarrollo Educativo \\ ISSN $2007-7467$}

En relación a las competencias ambientales de estudiantes y profesores, los estudiantes del programa educativo de Sudáfrica no promueven competencias, y los de México y de Brasil están casi en la misma situación, es decir, falta fortalecer sus competencias en este sentido. Debido a que manifiestan que, aunque hay temáticas ambientales en los programas, no son suficientes. En este orden de ideas, los maestros del programa de Sudáfrica manifestaron no contar con competencias en este campo, los de México consideran que no son suficientes y los de Brasil estipulan que cuentan con ellas. En referencia al análisis comparativo de la información proporcionada por informantes clave, referente a comprender la presencia de la EA en el currículo de los programas educativos, se evidenció que solo dos programas de la universidad de México y los dos de Brasil contemplan de alguna forma la EA. Sin embargo, la presencia de esta educación en aquella es en menor proporción y de manera disciplinar; mientras que, en esta, en la de Brasil, es en gran proporción su incorporación, aunque aún su integración de manera transversal está en proceso. En el programa de Sudáfrica, la EA no figura en el currículo del programa.

Las competencias de algunas unidades de aprendizaje o materias en los programas de México donde figura la EA se relacionan con las competencias específicas del plan de estudios, es decir, si en el programa de derecho se imparte legislación ambiental, solo se enfoca en el medio ambiente y su inserción en lo jurídico. Para que se implemente con enfoque sustentable se debe vincular con aspectos sociales, económicos, ambientales, naturales. En el caso del programa de Brasil, las competencias ambientales que se promueven se apegan a la sustentabilidad.

En el análisis comparativo del fundamento institucional o legal, en relación con establecimiento de la EA en el currículo de los programas educativos, se constató que los programas educativos de la UAGro tienen como sustento al Modelo educativo (UAGro, 2004) de la misma universidad, que es el documento que establece lineamientos curriculares sobre la educación que se brinda. Este establece que en todos los programas educativos de la universidad se implemente la EA como un eje transversal. El modelo educativo de la UAGro contempla como principio fundamental a la sustentabilidad y, por ende, las problemáticas ambientales que se viven en el mundo, en México y en el estado donde está inmersa la universidad. Ante tal situación, se establece como lineamiento curricular en los ejes transversales al medio ambiente. En el programa educativo de la universidad de Sudáfrica, no se tuvo acceso a algún documento, sin embargo, el informante clave aseguró que en el programa no se contempla la dimensión ambiental. En cambio, los programas educativos de Brasil tienen como sustento la "Resolución NN. 2, 15 de junio de 2012. Directrices Curriculares Nacionales para la Educación Ambiental del Ministerio de Educación, del Consejo Nacional de Educación de Brasil, la Resolución 053/2014/Rectoría, Universidad Regional 


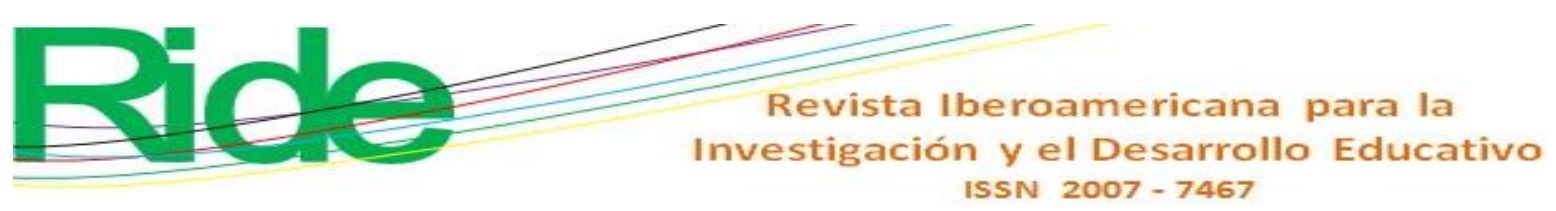

de Blumenau", que establece la necesidad de incorporar la EA curricularmente, y, por ende, los planes de estudio de las Licenciaturas en Biología y Arquitectura y Urbanismo establecen en su estructura curricular la dimensión de esta y de la sustentabilidad.

Comparando este estudio con el desarrollado por Pérez (2015), quien investigó sobre la incorporación de lo ambiental dentro del programa de Biología de la Universidad del Tolima en Colombia y quien concluyó que el componente ambiental no se encuentra allí incluido; en comparación con este trabajo, como se decía, en el caso de los programas educativos de la universidad de México y de la universidad de Brasil sí se hace presente de alguna forma, mientras que en el caso del programa de la universidad de Sudáfrica es escaso. Como se estipuló ya con el estudio de Pérez (2015), hay universidades que no integran la dimensión ambiental o ponen en práctica la EA, a pesar de las sugerencias mundiales que hacen referencia a la crisis ambiental, la cual sin duda amerita que las instituciones universitarias en el mundo contribuyan con la incorporación y propagación de los saberes ambientales, ya sea través de disciplinas o transversalmente en sus currículos.

\section{Conclusiones}

Los currículos universitarios no deben de estar desvinculados del contexto, ya que es ahí donde se identifican las diferentes problemáticas que se considerarán posteriormente como necesidades para ser integradas en el proyecto educativo a echar a andar. La problemática o crisis ambiental es de tipo local y global y, por consiguiente, es importante incluir esta dimensión. La EA surgió como una herramienta que viene a hacer conscientes a los seres humanos para cuidar y preservar los recursos que la naturaleza ofrece. No obstante, en los últimos años, se ha enfocado a despertar conciencias promoviendo conocimientos, habilidades, actitudes y valores con un enfoque no solo natural sino social, lo cual implica vincular estas problemáticas con aspectos económicos y políticos, entre otros, para que el ser humano contribuya con propuestas y acciones ante lo que sucede con el medio. Es así como los programas educativos en las universidades deben de integrar e implementar esta educación. Sin embargo, existen métodos para su inclusión curricular que van de los disciplinares hasta los transversales. Algunos autores en esta materia recomiendan los transversales, porque de esta forma se impregna todo el proyecto educativo y no solo una parte. 


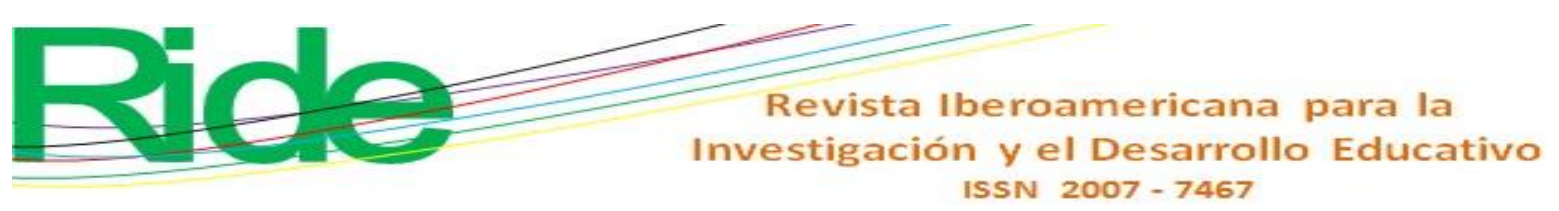

La EA es una estrategia viable para que las instituciones educativas a nivel mundial desde el nivel básico y hasta el nivel superior promuevan el cuidado del medio ambiente y, con ello, coadyuvar en la sustentabilidad. Se percibe que los programas educativos que incorporan la EA son los de la FURB, con un enfoque disciplinar y su inclusión transversal está en proceso; los programas de México, aunque integran en menor medida esta educación y de manera disciplinar, requieren ser fortalecidos en este campo. En el caso del programa sudafricano definitivamente se requiere ambientalizar, ya sea a través de una disciplina o con un enfoque transversal, debido a que fue el programa que no contempla la dimensión ambiental. En cuanto a los programas educativos de México y Brasil, su fortaleza de implementar la EA está en sus documentos institucionales y oficiales, ya que allí está establecida su incorporación. Respecto al programa educativo de Sudáfrica, aunque no se tuvo acceso a un documento institucional que establezca la incorporación de la EA, el informante clave lo confirma. Un soporte para incorporar la dimensión ambiental sería los resolutivos de reuniones internacionales como los de la ONU o el Pnuma, debido a que desde mediados del siglo pasado hacen alusión a que las problemáticas ambientales se agravan y extienden en el mundo y, por tal razón, la EA debe ser insertada en la educación. 


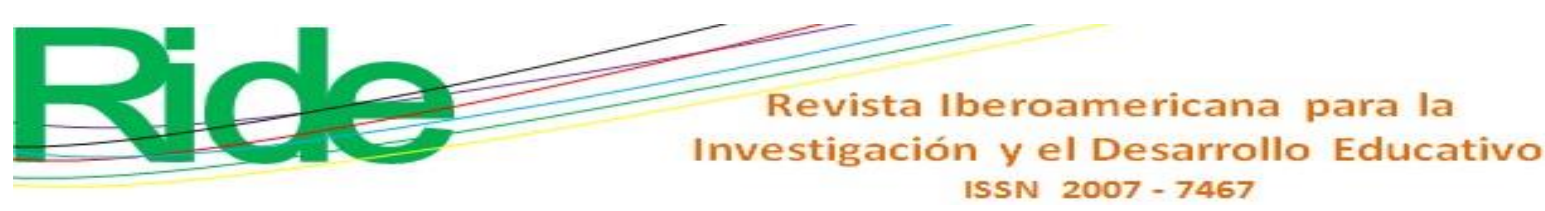

\section{Referencias}

Alonso, M. (2010). Historia de la educación ambiental: La educación ambiental en el siglo XX. España: Asociación Española de Educación Ambiental.

Batllori, G. A. (2008). La educación ambiental para la sustentabilidad: un reto para las universidades. Cuernavaca, México: Universidad Nacional Autónoma de México-Centro Regional de Investigaciones Multidisciplinarias. Recuperado de https://www.crim.unam.mx/web/sites/default/files/La\%20educaci\%C3\%B3n\%20ambient al\%20para\%20la\%20sustentabilidad.pdf.

Cantú-Martínez, P. (2014). Educación ambiental y la escuela como espacio educativo para la promoción de la sustentabilidad. Revista Electrónica Educare, 18(3), 39-52.

Conde, N. (2004). Integración de la educación ambiental en los centros educativos. Ecocentros de Extremadura: análisis de una experiencia de investigación-acción. (memoria de tesis doctoral). Universidad de Extremadura, Cáceres. Recuperado de https://dialnet.unirioja.es/descarga/tesis/176.pdf.

Eschenhagen, M.L. (2007). Las cumbres ambientales internacionales y la educación ambiental, $\begin{array}{llll}\text { OASIS, } & \text { (12), } & \text { 39-76. } & \text { Recuperado }\end{array}$ https://revistas.uexternado.edu.co/index.php/oasis/article/view/2412.

Galindo, G. L. (2015). La educación ambiental en la virtualidad: un acercamiento al estado del arte. RIDE Revista Iberoamericana para la Investigación y el Desarrollo Educativo, 5(10), 143. Recuperado de https://www.ride.org.mx/index.php/RIDE/article/view/195/860.

García, D. y Priotto, G. (2009). Educación ambiental: Aportes políticos y pedagógicos en la construcción del campo de la educación ambiental. Buenos Aires, Argentina: Secretaría de Ambiente y Desarrollo Sustentable.

Herrera, A. R. (2013). De la crisis a la ambientalización curricular. La trayectoria de la sustentabilidad. Revista Visión Educativa IUNAES, 7(15), 47-56. Recuperado de http://iunaes.mx/wp-content/uploads/2013/04/15-Abril-de-2013.pdf.

Kwesi, M. B. (2009). An overview of environmental issues in Southern Africa. African Journal of Ecology, 47(1), 93-98.

Macedo, B. y Salgado, C. (2007). Educación ambiental y educación para el desarrollo sostenible en América Latina. Revista de la Cátedra Unesco sobre desarrollo sostenible, (1), 29-37. Recuperado de http://www.ehu.eus/cdsea/web/wp-content/uploads/2016/12/Revista1.pdf. 


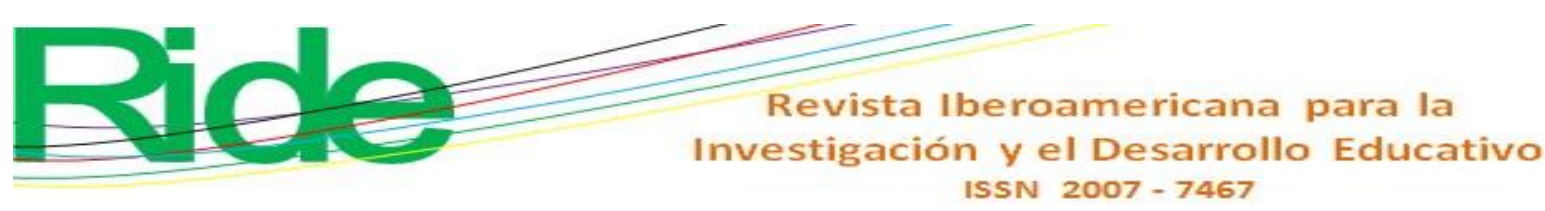

Rengifo, B., Quitiaquez, L. y Mora, F. (2012). La educación ambiental una estrategia pedagógica que contribuye a la solución de la problemática ambiental en Colombia. Ponencia presentada en el XII Coloquio de Geocrítica. Bogotá, del 7 al 11 de mayo de 2012.

Santiago, A. (2009). La globalización del deterioro ambiental. Aldea Mundo, 4(27), 63-72.

Tetreault, D., Ochoa, H. y Hernández, E. (coords.) (2012). Conflictos socioambientales y alternativas de la sociedad civil. Guadalajara, México: Iteso. Recuperado de https://rei.iteso.mx/bitstream/handle/11117/425/Conflictos\%5B1\%5D.pdf?sequence=2

Universidad Autónoma de Guerrero [UAGro]. (2013). Modelo educativo. Hacia una educación de calidad con inclusión social. México: Universidad Autónoma de Guerrero. Recuperado de http://ingenieria.uagro.mx/inicio/files/normativa/Modelo_Educativo_de_la_UA Gro.pdf

Universidad Autónoma de Guerrero [UAGro]. (2014a). Anuario Estadístico UAGro 2013-2014. México: Universidad Autónoma de Guerrero. Recuperado de informacionestadistica.uagro.mx/anuarios/Anuario_Estadistico_UAGro_2013-2014.pdf

Universidad Autónoma de Guerrero [UAGro]. (2004b). Modelo Educativo. Chilpancingo, México: Universidad Autónoma de Guerrero.

Universidad Autónoma de Guerrero [UAGro]. (2015). Anuario Estadístico UAGro 2014-2015. México: Universidad Autónoma de Guerrero. Recuperado de informacionestadistica.uagro.mx/anuarios/Anuario_Estadistico_UAGro_2014-2015.pdf.

Universidad Regional de Blumenau [FURB]. (2012b). Resolución No. 2, 15 de junio de 2012. Brasil: Universidad Regional de Blumenau.

Universidad Regional de Blumenau (2014). Resolución 053/2014/Rectoría. Brasil: Universidad Regional de Blumenau.

Zabalza, B. M. (2012). Territorio, cultura y civilización. Interacções, (22), 6-33. Recuperado de https://revistas.rcaap.pt/interaccoes/article/view/1534/1225.

Zabala, G. I. y García, M. (2008). Historia de la Educación Ambiental desde su discusión y análisis en los congresos internacionales. Revista de Investigación, (63), 201-218. Recuperado de https://dialnet.unirioja.es/descarga/articulo/2547197.pdf. 


\begin{tabular}{|c|c|}
\hline Rol de Contribución & Autor (es) \\
\hline Conceptualización & Ramón Bedolla Solano \\
\hline Metodología & $\begin{array}{l}\text { Ramón Bedolla Solano «principal» Adriana Miranda Esteban «igual» Juan José } \\
\text { Bedolla Solano «igual» }\end{array}$ \\
\hline Software & $\begin{array}{l}\text { Ramón Bedolla Solano «principal» Adriana Miranda Esteban «igual» Juan José } \\
\text { Bedolla Solano «igual» }\end{array}$ \\
\hline Validación & $\begin{array}{l}\text { Ramón Bedolla Solano «principal» Adriana Miranda Esteban «igual» Juan José } \\
\text { Bedolla Solano «igual» }\end{array}$ \\
\hline Análisis Formal & Ramón Bedolla Solano «principal» Adriana Miranda Esteban \\
\hline Investigación & $\begin{array}{l}\text { Ramón Bedolla Solano «igual» Adriana Miranda Esteban «igual» Oscar Sánchez } \\
\text { Adame «igual» Juan José Bedolla Solano «igual» }\end{array}$ \\
\hline Recursos & Ramón Bedolla Solano «principal» Adriana Miranda Esteban \\
\hline Curación de datos & Ramón Bedolla Solano \\
\hline $\begin{array}{l}\text { Escritura - Preparación del borrador } \\
\text { original }\end{array}$ & Ramón Bedolla Solano \\
\hline Escritura - Revisión y edición & $\begin{array}{l}\text { Ramón Bedolla Solano «principal» Adriana Miranda Esteban «igual» Oscar } \\
\text { Sánchez Adame «igual» Juan José Bedolla Solano «igual» }\end{array}$ \\
\hline Visualización & $\begin{array}{l}\text { Ramón Bedolla Solano «principal» Adriana Miranda Esteban «igual» Oscar } \\
\text { Sánchez Adame «igual» Juan José Bedolla Solano «igual» }\end{array}$ \\
\hline Supervisión & $\begin{array}{l}\text { Ramón Bedolla Solano «principal» Adriana Miranda Esteban «igual» Oscar } \\
\text { Sánchez Adame «igual» Juan José Bedolla Solano «igual» }\end{array}$ \\
\hline Administración de Proyectos & Ramón Bedolla Solano \\
\hline Adquisición de fondos & Ramón Bedolla Solano \\
\hline
\end{tabular}

